\title{
Effect of cornet device to clear the excess trachea bronchial secretions in a 45 year old right middle and lower lobe pneumonia patient- a case report
}

\begin{abstract}
A 45- year old woman diagnosed as right middle and lower lobe pneumonia had been referred for chest physiotherapy to evacuate secretions and to improve oxygenation. The X-ray and Computed tomography findings revealed patchy areas of collapse, consolidation and fibrotic strands in right middle and lower lobes. The physical examination findings also confirmed the signs of consolidation and collapse i.e. decreased air entry on right side and presence of crepitations. Chest physiotherapy mainly aimed to clear the excess trachea bronchial secretions in pneumonia. Chest physiotherapy with the help of positive expiratory device -Cornet was given twice daily for ten days. Cornet device uses the principle of positive expiratory pressure which creates an airway oscillation inside the airways. This causes thinning down of mucus and the patient can expectorate the secretions with the help of cough or huff. The X-ray findings showed improvement in right lower lobe and there was no radiological evidence of consolidation and collapse post chest physiotherapy. The patient responded to treatment well and she was discharged successfully. It is concluded that the present case study advocates the use of Cornet device to clear the excess tracheobronchial secretions. Cornet device is easy to use without any supervision and the patient may use at home also.
\end{abstract}

Volume I Issue I - 2016

\author{
T S Muthukumar,' MohanKumar \\ Thekkinkattil ${ }^{2}$ \\ 'Departement of Physiotherapy, SRIPMS, India \\ ${ }^{2}$ Sri Ramakrishna Hospital, India
}

\begin{abstract}
Correspondence: TS Muthukumar, Professor, College of Physiotherapy SRIPMS, 395 Sarojini Naidu Road, New Sidhhapudur, Coimbatore-64I044, Tamilnadu, India, Tel +91-4224500I63, Fax +91-422-4500193,

Email muthukumarphysio@gmail.com
\end{abstract}

Received: July 20, 2016 | Published: July 29, 2016

Keywords: pneumonia, chest physiotherapy, collapse, consolidation

\section{Introduction}

Pneumonia is a common respiratory infection in the community that can affect people of all ages. The incidence increases markedly among the elderly, and, with an aging population, it will become an increasingly important disease. The annual incidence of pneumonia in the elderly is between 25 to 44 per a population of 1000 for non institutionalized patients and four times that of patients younger than $65 .{ }^{1}$ For the elderly in residential care, the incidence increases to 33 to 114 cases per population of 1000 per year. ${ }^{1}$ The main indication for chest physiotherapy in Pneumonia is to evacuate inflammatory exudates in a patient whose airways or gas exchanging tissues are pathologically affected by a microbial infection. ${ }^{2}$ Research studies proved efficacy of chest physiotherapy in Pneumonias but there are limited clinical trials for Cornet device.

Positive expiratory devices such as Acapella, Flutter, Cornet, and Quake are widely used in clinical settings to increase the efficiency of airway clearance. Positive expiratory pressure devices were introduced in the 1970s and each device has its own merits and demerits. ${ }^{4}$ Positive expiratory pressure devices work on the concept of active expiration through a one way valve against a variable resistance. Flow resistance can be manipulated to adjust for a desired pressure. The clinical effects of PEP devices are to enhance the secretion removal, to help prevent infections, to help mitigate atelectasis and to improve pulmonary mechanics and gas exchange. The possible underlying mechanism of PEP devices are: it fills the under-inflated or collapsed alveoli via collateral ventilation (pores of Kohn, Canals of Lambert) and it helps to stint the airways open during expiration. ${ }^{5}$
Cornet is one of the positive expiratory devices consists of a semicircular tube containing a flexible latex-free hose. Expiration through the device causes the hose to flex, buckle and unbuckle leading to oscillating positive pressure in the patient airways which fluctuates many times per second. The mouth piece can be adjusted to generate the desired effect. Unlike Flutter the cornet is gravity independent and can be employed in any position. In to Her study we analyzed the effect of cornet device in a single pneumonia patient.

\section{Case description}

A case of 45 -year old woman diagnosed as Pneumonia admitted in male pulmonology ward at Sri Ramakrishna Hospital. The patient came with a history of fever with chills and rigors, cough with cold, vomiting, sweating of legs and facial sweating. At the time of admission the vital signs were stable with Saturation of percentage of oxygen $\left(\mathrm{SpO}_{2}\right)$ is $86 \%$ without oxygen therapy. The patient had history of similar episodes on and off treated at local hospital nearby. The personal history revealed the habit of chewing betel nuts. The patient had no history of coexisting morbidities.

On physical examination, pedal edema, facial swelling was present, but no signs of anemia and clubbing. On examination her dyspnea level was 3 according to MRC grading. Her rate of perceived exertion according to rate of perceived exertion was 13.The vital signs were Heart rate 90 beats per minute, respiratory rate-18 breaths/minute, and temperature was $100^{\circ} \mathrm{F}$. Patient's chest expansion was reduced at nipple level. Pulmonary function test had not been taken due to acute infection. On auscultation Heart sounds S1 and S2 heard and presence of bilateral crepitations but more on right middle and lower lobe. She 
had productive cough more at mornings and quantity of sputum was measured using sputum weight and it showed $35 \mathrm{~g}$. The color of the sputum was yellow and the consistency was mucopurulent.

The microbiological studies of throat swab showed no growth of pathogens and gram staining reports showed negative for Acid Fast Bacilli. The peripheral smear showed moderate hypochromic anaemia. Montoux test was negative after 48 hours. The malaria cord test and the dengue rapid test showed negative results. The echocardiogram reports revealed restrictive cardiomyopathy. The X-ray and Computed tomography findings revealed patchy areas of collapse, consolidation and fibrotic strands in right middle and lower lobes with enlarged lymph nodes and minimal right pleural effusion. Based on the physical findings, other investigations and differential diagnosis the patient had been diagnosed as Right lower lobe pneumonia. He was treated with broad spectrum antibiotics to combat infections and also levolin nebulisation $8^{\text {th }}$ hourly for 7 days. ${ }^{6}$

\section{Outcomes}

Chest physiotherapy is an important treatment strategy to evacuate the excess tracheobronchial secretions from the patient's right lower lobe and middle lobe. Chest physiotherapy comprises of bronchial hygiene techniques such as percussion, vibration, suctioning and nebulisation and also postural drainage which helpful in draining the secretions into the proximal airways. Positive expiratory devices such as Flutter, Acapella Cornet are used routinely as an adjunct form of chest physiotherapy. But the effect of RC Cornet device was not adequately reported. Based on the evidence based practice which strongly advocates the use of chest physiotherapy to clear excess trachea bronchial secretions from the Pneumonia affected lungs and thereby improves the oxygenation and ventilations of the lungs.

We had started giving chest physiotherapy treatment with the use of RC Cornet device from the day of referral by asking the patient to actively expire through the mouthpiece of cornet and it created a buckling and unbuckling of hose which in turn produced a back pressure and caused the airway oscillation inside the patient's airways. Patient was asked to do tHer technique for 3-5 minutes for each cycle with 5 minutes rest periods and for about 15 minutes twice daily with supervision. After 15 minutes the patient was asked to actively cough or huff to evacuate the secretions. The treatment continued for about 10 days.

With the use of RC Cornet (Figure 1) along with conventional chest physiotherapy the patient improved better when compared to the day of admission and it was evident subjectively. The quantity of the sputum was reduced since the weight of the sputum mug was 0.5 $\mathrm{g}$ and her respiratory rate was 16 breaths per minute post treatment. The perception of dyspnea was reduced due to improvement in gaseous exchange since the secretions block the air passages. After the treatment, the rate of perceived exertion according to Borg scale was 9. On auscultation there were no crepitations and air entry was equal. The percentage of saturation of oxygen was $100 \%$ room air. There was also good improvement in radiological findings before and after the treatment and it was shown in Figures $2 \& 3$ respectively. When we compared the X-ray findings during the time of admission and 10 days after the treatment, consolidation was markedly reduced and the patient responded well for the treatment.

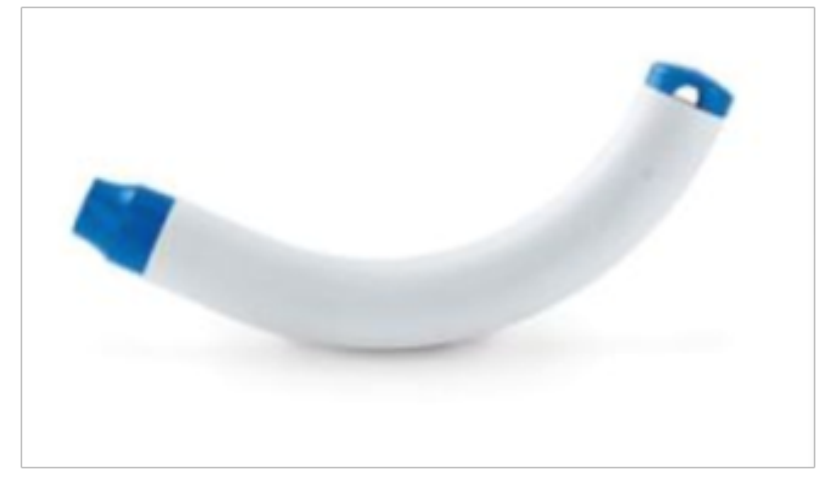

Figure I RC-cornet devices.

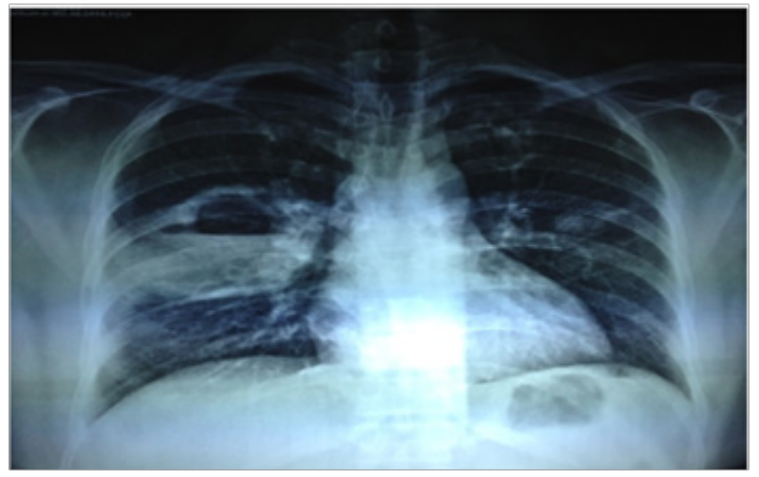

Figure 2 Chest $\mathrm{X}$-ray at the time of admission.

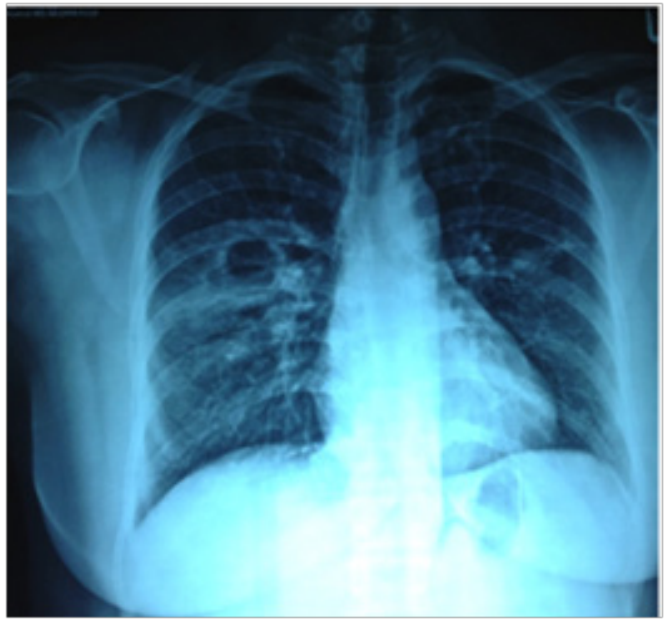

Figure 3 Chest $X$-ray post treatment.

\section{Discussion}

Good chest physiotherapy reduces the length of the stay in hospital for pneumonia patients. RC Cornet works on the principle of positive pressure oscillating at three frequencies. The low frequency measures at $20 \mathrm{~Hz}$, middle frequency measures at $80 \mathrm{~Hz}$ and high frequency oscillation of $300 \mathrm{~Hz}$. The oscillating pressure dilates the bronchi and bronchioles, moves the mucus from the bronchial walls and decreases mucous viscosity. On blowing the device the hose is forced into two compartments with a flexible valve at each end. 
During exhalation, the critical pressure of the first valve exceeds, then the air enters the second compartment and it opens the valve while the first valve closes. It causes a positive pressure with some pressure fluctuations inside the airways. The mouth piece can be altered into different positions which cause the twisting of hose and it increases the amplitude of pressure oscillation. The airflow oscillations cause more vibration of mucus molecules and transports from distal to proximal. It also reduces the visco- elasticity of the mucus and it makes thinner. In comparison to manual techniques of airway clearance, the Cornet device has the advantage of vibrating most distal airways through pores of Kohn and it ruptures the cross linking bonds such as the disulphide bridges and conversion of macro molecules to micro molecules. Based on the study of Amit Vyas et al 2012, this case report also showed favor of using Cornet device in superior with manual airway clearance techniques to clear the excess tracheobronchial secretions in right middle and lower lobe pneumonia patient as it was evident in chest x-ray. The future study may incorporate many patients and other parameters may also be included.

\section{Conclusion}

It is concluded that the present case study advocates the use of Cornet device to clear the excess tracheobronchial secretions. Cornet device is easy to use without any supervision and the patient may use at home also.

\section{Ethical committee approval}

This case study has been approved by the institutional review board of College of Physiotherapy S.R.I.P.M.S Coimbatore Tamilnadu India.

\section{Acknowledgements}

None.

\section{Conflict of interest}

The author declares no conflict of interest.

\section{References}

1. Janssens JP, Krause KH. Pneumonia in the very old. Lancet Infect Dis. 2004;4(2):112-124.

2. Britton $\mathrm{S}$, Bejstedt $\mathrm{M}$, Vedin L. Chest physiotherapy in primary pneumonia. Br Med J (Clin Res Ed). 1985;290(6483):1703-1704.

3. Okeson CD, Paul M. Performance characteristics of three hand held airway clearance devices: Quake, Acapella, Flutter. Respirology. 2006;51(11):1314.

4. Allen K, Cariters C. Airway clearance modality and devices. American academy of nurse practitioners; 2009.

5. Cegla UH, Jost HJ, Harten A, et al. Rc- cornet improves the efficacy of inhalation therapy with ipratropiumbromide in COPD- patients. Pneumologie. 2011;55:465-469.

6. Amit Vyas A, Vaishali K, Gopal Krishna A, et al. Comparison of quake and RC-cornet for airway clearance in bronchiectasis: A randomized cross over trial. IJHSR. 2012;2(6):1-8. 